\title{
Successful rapid subcutaneous desensitization to anakinra in a case with a severe immediate-type hypersensitivity reaction
}

Erciyes University School of Medicine, Department of Chest Diseases, Division of Allergy and Clinical Immunology, Kayseri, Turkey

\section{KeY WORDS}

drug allergy; immediate hypersensitivity;

interleukin-1 receptor antagonist;

anakinra; immunologic desensitization

\section{Corresponding author}

Murat Türk

Erciyes University Hospital

Division of Allergy and Clinical Immunology

38039 Melikgazi, Kayseri, Turkey

Phone: +90352 20766 66, 21180

E-mail: mrttrk@gmail.com

\section{Doi}

10.23822/EurAnnACI.1764-1489.30

\begin{abstract}
Summary
Anakinra, one of the novel biological agents, is a recombinant human IL-1 receptor antagonist. It is preferred as an alternative drug for familial Mediterranean fever cases where colchicine is not sufficient or cannot be used due to its side effects. Like all other biologics, hypersensitivity reactions to anakinra are quite rare. This is the first case which was successfully desensitized with anakinra after a severe immediate-type hypersensitivity reaction.
\end{abstract}

\section{Introduction}

Anti-interleukin (IL)-1 agents are one of the biological agents that have increased use in recent years. The anti-IL-1 agent anakinra is a recombinant human IL-1 receptor antagonist that is used as a treatment option in various disorders (1). Despite a good safety profile, local reactions at the injection site are common (2-4). Familial Mediterranean fever (FMF) is an autosomal recessive inflammatory disease in which colchicine is used as the primary treatment (5-7). Anakinra is an alternative drug for FMF cases where colchicine is not sufficient, or colchicine cannot be used due to its side effects (7). Systemic reactions are quite rare with anti-IL-1 agents and they are generally well tolerated $(8,9)$. We present a 38 -year-old female who developed an immediate-type hypersensitivity reaction after the use of anakinra and then underwent successful desensitization.

\section{Case presentation}

The patient was referred to our clinic by the Department of Rheumatology due to a history of anaphylaxis after the use of anakinra. Her prior medical history revealed that after the diagnosis of FMF a year before, she was prescribed with colchicine at first. However, she had developed nausea, vomiting, cough, dyspnea, wheezing, dysphagia, and fatigue within 30 minutes after ingestion of the first colchicine dose. She had rapid recovery within one hour of treatment, but at the emergency room she was not able to remember the names of the drugs taken. She retried colchicine on her own a week later, and presented to emergency room again with the same reactions. Anakinra treatment was started by the rheumatology department, due to the history of immediate-type hypersensitivity reaction with colchicine and newly diagnosed renal amyloidosis. She again developed vomiting and nausea, dyspnea, 
Table I - Protocol for subcutaneous desensitization with anakinra ${ }^{l}$.

\begin{tabular}{ccccc}
\hline Steps $^{\mathbf{2}}$ & Dilution & Volume $(\mathbf{m L})$ & Injected dose (mg) & Cumulative dose (mg) \\
\hline 1 & $1 / 1000$ & 0.1 & 0.015 & 0.015 \\
\hline 2 & & 0.3 & 0.045 & 0.06 \\
\hline 3 & & 0.6 & 0.09 & 0.15 \\
\hline 4 & $1 / 100$ & 0.1 & 0.15 & 0.3 \\
\hline 5 & & 0.3 & 0.45 & 0.75 \\
\hline 6 & $1 / 10$ & 0.6 & 0.9 & 1.65 \\
\hline 7 & & 0.3 & 3 & 4.65 \\
\hline 8 & $1 / 1$ & 0.6 & 6 & 10.65 \\
\hline 9 & & 0.1 & 10 & 20.65 \\
\hline 10 & & 0.25 & 25 & 45.65 \\
\hline 11 & & 0.55 & 55 & 100.65 \\
\hline
\end{tabular}

${ }^{1}$ Premedication with $5 \mathrm{mg}$ of desloratadine and methyl-prednisolone $40 \mathrm{mg}$ was administered 30 minutes before the desensitization procedure.

${ }^{2}$ Administered at 30-minute intervals.

cough, wheezing, and dysphagia 5 minutes after the first dose of subcutaneous injection of anakinra (Kineret ${ }^{\circledR} 0.67 \mathrm{~mL}[100$ $\mathrm{mg}$; Sobi Inc., Stockholm, Sweden). She was treated with methylprednisolone, pheniramine maleate and oxygen therapy. Her symptoms relieved within one hour.

The patient was referred to our clinic for drug allergy evaluation and desensitization. The results of skin prick and intradermal tests with the undiluted form of Kineret ${ }^{\circledR}$ were all negative. Desensitization with anakinra was planned due to nonexistence of an alternative drug. Although there was no doubt about the typical hypersensitivity reaction, a single blind placebo challenge was performed with physiological saline prior desensitization procedure, to rule out the possibility of a psychosomatic basis. No reaction was observed with the placebo challenge (3 different physiological saline doses of $0.1 \mathrm{cc}, 0.5 \mathrm{cc}$, and $1 \mathrm{cc}$ administered SC with 30 minute intervals). Premedication with desloratadine oral tablet and methylprednisolone $40 \mathrm{mg}$ IV was administered one hour before the desensitization procedure. The initial concentration for the desensitization was $0.1 \mathrm{ml}$ of a 1/1000 dilution of the therapeutic dose $(100 \mathrm{mg}=0.67 \mathrm{ml})$. Cumulative dosing was achieved by SC administrations of increasing doses at 30-minute intervals (table I). The desensitization protocol was completed without any reaction. One week later, total injection dose was divided into two and administered SC into separate arms, as local erythema and edema (about $10 \mathrm{~cm}$ ) developed at the injection site. The patient was able to use anakinra without premedication and without any problems after the desensitization procedure.

\section{Discussion}

Interleukin-1 antagonists have been recently used, beyond rheumatoid arthritis, in different disorders, such as FMF $(4,6)$. Anakinra is a safe drug that is generally well tolerated. Local reactions at the injection site are frequently reported, but systemic reactions are quite rare $(4,8-10)$. As far as we know, this is the second case that developed a severe immediate-type hypersensitivity reaction and successfully desensitized with anakinra.

The role of an IgE-mediated mechanism in anaphylaxis is a controversial issue in the instance that a patient has never been exposed to a drug before. On the other hand, some studies show that previous contact with the causative drug is not an obligatory prerequisite for immune-mediated drug hypersensitivity, and cross-reactivity between the involved drug and unrecognized prior exposure to similar chemical structures cannot be ruled out (11). Our patient did not have a prior history of anakinra usage and her skin tests were negative. The timing and type of reaction strongly suggest a non-IgE-mediated immediate-type hypersensitivity mechanism to anakinra.

Desensitization is a safe method of re-administering a drug that causes immediate-type hypersensitivity reactions (12). Desensitization with anakinra has previously been reported in four separate case reports in the literature $(10,13-15)$. Three of these cases were desensitized for a late-onset hypersensitivity reaction, and one case was desensitized for an immediate hypersensitivity reaction with anakinra (13-15). In the first reported case with an immediate-type hypersensitivity reaction (urticaria + angioedema) sensitization to anakinra was shown with skin tests, and the patient was successfully 
desensitized (10). However, the reaction was thought to be a mild systemic reaction due to the presence of skin and mucosa findings only. Desensitization was started with $0.1 \mathrm{ml}$ of a $1 / 100$ dilution of anakinra, since starting with higher doses was recommended for cases with mild systemic reactions. Since the reaction intensity of our case was more severe, desensitization was started with more diluted doses and the protocol was completed without any reactions. In conclusion, this is the first case which was successfully desensitized with anakinra after a severe immediate-type hypersensitivity reaction to anakinra. Desensitization can be performed using this protocol in cases with a severe systemic reaction. Total daily dose may be divided into two and administered SC into separate arms, if local reactions develop during follow-up.

\section{Patient consent}

Obtained.

\section{Conflict of interest}

The authors declare that they have no conflict of interest.

\section{References}

1. Vitale A, Insalaco A, Sfriso P et al. A Snapshot on the on-label and off-label use of the interleukin-1 inhibitors in Italy among rheumatologists and pediatric rheumatologists: a nationwide multi-center retrospective observational study. Front Pharmacol 2016; 7:380.

2. Emmi G, Silvestri E, Squatrito D et al. Long-term efficacy and safety of anakinra in a patient with Behcet's disease and concomitant tuberculosis infection. Int J Dermatol 2017; 56:218-20.

3. Kaiser C, Knight A, Nordström D et al. Injection-site reactions upon Kineret [anakinra] administration: experiences and explanations. Rheumatol Int 2012; 32:295-9.
4. Vila AT, Puig L, Fernández-Figueras MT et al. Adverse cutaneous reactions to anakinra in patients with rheumatoid arthritis: clinicopathological study of five patients. Br J Dermatol 2005; 153:417-23.

5. Meinzer U, Quartier P, Alexandra J et al. Interleukin-1 targeting drugs in familial Mediterranean fever: a case series and a review of the literature. Semin Arthritis Rheum 2011; 41:265-71.

6. Grattagliano I, Bonfrate L, Ruggiero V et al. Novel therapeutics for the treatment of familial Mediterranean fever: from colchicine to biologics. Clin Pharmacol Ther 2014; 95:89-97.

7. Alpay N, Sumnu A, Çalışkan Y et al. Efficacy of anakinra treatment in a patient with colchicine-resistant familial Mediterranean fever. Rheumatol Int 2012; 32:3277-9.

8. Desai D, Goldbach-Mansky R, Milner JD et al. Anaphylactic reaction to anakinra in a rheumatoid arthritis patient intolerant to multiple nonbiologic and biologic disease-modifying antirheumatic drugs. Ann Pharmacother 2009; 43:967-72.

9. Anton J, Ros J, Ricart $S$ et al. Desensitization to anakinra in a systemic onset juvenile idiopathic arthritis patient [abstract P-079]. Clin Exp Rheumatol 2005; 23(suppl 37):24.

10. Şoyyiğit S, Kendirlinan R, Aydın O et al. Successful desensitization with anakinra in a case with immediate hypersensitivity reaction. Ann Allergy Asthma Immunol 2014; 113:325-6.

11. Schnyder B. Approach to the patient with drug allergy. Immunol Allergy Clin N Am 2009; 29:405-18.

12. Cernadas JR, Brockow K, Romano A et al. General considerations on rapid desensitization for drug hypersensitivity consensus statement. Allergy 2010; 65:1357-66.

13. Emmi G, Silvestri E, Cantarini L et al. Rapid desensitization to anakinra-related delayed reaction: need for a standardized protocol. J Dermatol 2016 Sep 26. doi: 10.1111/1346-8138.13619. [Epub ahead of print]

14. Leroy V, Lazaro E, Darrigade AS et al. Successful rapid subcutaneous desensitization to Anakinra in a case of delayed-type hypersensitivity reaction. Br J Dermatol 2016; 174:1417-8.

15. Verduga MZ, Tavera UMA, Pereira DA et al. Desensitization to anakinra: case report. J Allergy Clin Immunol 2014; 133:AB274. 